\title{
GRUPO DE APOIO/SUPORTE COMO ESTRATÉGIA DE CUIDADO: IMPORTÂNCIA PARA FAMILIARES DE USUÁRIOS DE DROGAS
}

\author{
Simone Quadros ALVAREZ ${ }^{a}$, Giovana Calcagno GOMES \\ Adriane Maria Netto de OLIVEIRAc ${ }^{\mathrm{c}}$, Daiani Modernel XAVIER ${ }^{\mathrm{d}}$
}

\section{RESUMO}

Objetivou-se conhecer a percepção de familiares de usuários de drogas acerca da importância do grupo de apoio/ suporte como estratégia de cuidado. Realizou-se uma pesquisa descritiva com abordagem qualitativa, no segundo semestre de 2010, em um Centro de Atenção Psicossocial para usuários de álcool e outras drogas (CAPS AD), de uma cidade do extremo sul do Brasil, com dez familiares. Os dados foram coletados através da entrevista semiestruturada e analisados pela Análise Temática. Verificou-se que o grupo é uma alternativa à internação no hospital psiquiátrico, possibilita acesso e acompanhamento pelos profissionais da saúde, ajuda na compreensão do que é a dependência química, instrumentaliza para o cuidado e possibilita a reinserção social do dependente químico. Conclui-se que o grupo de apoio/suporte se constitui em relevante estratégia de cuidado aos familiares de usuários de drogas, apresentando-se como um instrumento a ser utilizado pelo enfermeiro em sua prática cotidiana.

Descritores: Família. Transtornos relacionados ao uso de substâncias. Cuidados de enfermagem.

\section{RESUMEN}

El objetivo fue investigar la percepción de los familiares de los usuarios de drogas sobre la importancia del grupo de apoyo/ soporte como una estrategia de atención. Se realizó un estudio descriptivo con enfoque cualitativo, en el segundo semestre de 2010, en un Centro de Atención Psicosocial para usuarios de alcohol y otras drogas (CAPS AD), en una ciudad del extremo sur de Brasil, con diez familiares. Los datos fueron recolectados por entrevistas semiestructuradas y analizados por el análisis temático. El grupo de apoyo/soporte es una alternativa a la hospitalización, puede proporcionar la supervisión periódica por profesionales de la salud, ayuda a entender qué es la adicción, prepara para el cuidado y permite la reinserción social del adicto. Se concluye que el grupo de apoyo/soporte es una estrategia para el cuidado de las familias. Se presenta como una tecnología para ser incorporada por las enfermeras en su práctica diaria.

Descriptores: Familia. Trastornos relacionados con sustancias. Atenciones de enfermería.

Título: Grupo de apoyo/soporte como estrategia de atención: importancia para familiares de usuarios de drogas.

\section{ABSTRACT}

The objective was to investigate the perception by relatives of drug users about the importance of the support group as a strategy of care. We conducted a descriptive study with qualitative approach, in the second half of 2010, in a psychosocial care center for users of alcohol and other drugs (CAPS AD), in a city in the extreme south of Brazil, with ten relatives. Data were collected by semistructured interviews and analyzed by thematic analysis. It was found that the group is an alternative to hospitalization in a psychiatric hospital, can provide access and regular monitoring by health professionals, helps understand what addiction is, provides tools for care and allows for the social rehabilitation of the addict. The support group was concluded to be an important strategy for caring for relatives of drug users, presenting itself as a technology to be incorporated by nurses in their daily practice.

Descriptors: Family. Substance-related disorders. Nursing care.

Title: Support group as a strategy of care: the importance for relatives of drug users.

a Enfermeira. Mestranda do Programa de Pós-graduação em Enfermagem da Universidade Federal do Rio Grande - FURG. Membro do Grupo de Estudos e Pesquisas em Enfermagem e Saúde da Criança e do Adolescente - GEPESCA/ FURG. simone.alvarez@ibest.com.br, Rio Grande/ RS/ Brasil.

b Enfermeira. Doutora em Enfermagem. Professora da Escola de Enfermagem da FURG. Líder do GEPESCA/ FURG. giovanacalcagno@, furg.br, Rio Grande/ RS/ Brasil.

c Doutora em Enfermagem. Docente da Escola de Enfermagem da FURG. Membro Grupo de Estudos e Pesquisa em Família, Enfermagem e Saúde-GEPEFES/ FURG. adrianeoliveira@furg.br, Rio Grande/ RS/ Brasil.

d Acadêmica da nona série do Curso de Enfermagem da FURG. Membro do GEPESCA/ FURG. daiamoder@ibest.com.br, Rio Grande/ RS/Brasil. 


\section{INTRODUÇÃO}

A dependência química vem mobilizando o sistema de saúde e ganhando visibilidade devido à complexidade na solução desse problema. Um número considerável de pessoas residentes em centros urbanos, tanto no Brasil, como no mundo, consomem de forma abusiva substâncias psicoativas ${ }^{(1-2)}$. No Brasil, estima-se que existam em torno de 900 mil usuários dessas substâncias, ocupando posição estratégica no combate ao tráfico internacional ${ }^{(3)}$.

Constata-se que, além de uma doença, a dependência química é um grave problema de saúde pública necessitando da atuação em busca de estratégias para a prevenção, o acompanhamento e o tratamento dos usuários e familiares. Os Centros de Atenção Psicossocial a usuários de álcool e outras drogas (CAPS AD) apresentam-se como uma destas estratégias. Atuam em toda a área territorial de referência e um de seus desafios consiste em descentralizar a assistência promovendo articulação social e intersetorial, buscando o estreitamento dos laços entre o campo da saúde mental e a comunidade ${ }^{(4)}$.

As atividades dos grupos de apoio/suporte com usuários de drogas e com familiares desenvolvidas nos CAPS AD apresentam-se como metodologias assistenciais utilizadas pelos profissionais da saúde/enfermagem com objetivo terapêutico e ferramenta de cuidado. Como base teórica para a abordagem grupal utilizou-se Loomis ${ }^{(5)}$ que apresenta o grupo de apoio/ suporte como aquele que tem por objetivo auxiliar pessoas durante períodos de ajustamento a mudanças, no tratamento de crises ou na manutenção ou adaptação a novas situações.

A tecnologia de grupo de apoio/ suporte é um recurso que vem sendo usado por profissionais de saúde, pois os auxilia a aliviar sentimentos de solidão e isolamento social, possibilitando troca de experiências e reflexão. A utilização de grupos de suporte/apoio requer a criação de um ambiente em que seus integrantes possam compartilhar suas experiências e sentimentos com a certeza de serem compreendidos pelos outros participantes ${ }^{(6)}$.

Ao oferecer apoio emocional e informações/ orientações, estes grupos possibilitam a percepção da situação real que estão vivendo, por meio do conhecimento de dados mais concretos sobre o problema e diminuição das fantasias a ele relacionadas, ajudando-os no enfrentamento da crise vivenciada. O grupo de apoio/ suporte oportuniza aprender novos comportamentos em clima de comparti- lhamento e aceitação. Por isso, apresenta-se como um excelente recurso terapêutico para lidar com pessoas que vivem situações de crise, tendo como objetivos promover coesão e apoio, elevando a autoestima e a autoconfiança de seus participantes ${ }^{(6)}$.

É uma forma de cuidar que favorece a interação e a integração de seus participantes, contribuindo para o processo de aprendizagem e de crescimento pessoal demandando habilidades em técnicas interpessoais de comunicação, relacionamento terapêutico e manejo grupal por parte do enfermeiro ${ }^{(7)}$. Além disso, o atendimento em grupo com participantes que vivenciam a mesma situação facilita a identificação, a troca de confidências, particularidades e intimidades entre os membros, e possibilita à equipe de saúde conhecer as reais necessidades e anseios desses familiares, facilitando o planejamento de uma assistência mais focada na família ${ }^{(8-9)}$. A atividade grupal é importante para o usuário de drogas em tratamento e para seu familiar auxiliando-os a conviverem com os problemas, aprendendo a manejá-los de modo mais saudável ${ }^{(10)}$.

O papel do enfermeiro, no grupo de apoio/ suporte, durante a atividade grupal com a família do usuário de drogas é a escuta, estabelecendo o processo de comunicação e relacionamento terapêutico. Através desta ferramenta de cuidado pode-se humanizar a assistência, estimulando o dependente químico e seu familiar a realizarem o enfrentamento das dificuldades e a manutenção do funcionamento psicossocial, de acordo com as necessidades de cada pessoa, a fim de fazê-la construir um novo projeto de vida e manter-se saudável ${ }^{(11)}$.

Por entender a família como essencial no processo terapêutico do usuário de drogas, a questão que norteou este estudo foi: qual a importância do grupo de apoio/ suporte como estratégia de cuidado, para familiares de usuários de drogas atendidos em um CAPS AD? A partir desta, o objetivo do estudo foi conhecer a percepção de familiares de usuários de drogas acerca da importância do grupo de apoio/ suporte como estratégia de cuidado.

\section{MÉTODOS}

O presente estudo é uma pesquisa descritiva com abordagem qualitativa. A pesquisa descritiva descreve o fenômeno investigado, possibilitando conhecer os problemas vivenciados ${ }^{(12)}$. É de abordagem qualitativa porque trabalha com um universo de significados, motivos, aspirações, crenças, valo- 
res e atitudes, permitindo maior profundidade das relações, dos processos e dos fenômenos que não podem ser traduzidos por meio de sua redução à operacionalização de variáveis ${ }^{(13)}$.

Foi desenvolvido no segundo semestre de 2010, em um CAPS AD de uma cidade do sul do país. O CAPS AD foi criado em novembro de 2009 e realiza grupos de usuários de drogas e de familiares, além de outras atividades preconizadas pelas políticas públicas referentes ao álcool e outras drogas. Estes ocorrem diariamente e são abertos. Há três salas para atividades em grupos e são realizados nos períodos da manhã e tarde.

Participaram do estudo dez familiares de usuários de drogas que frequentam as atividades grupais. Todos assinaram o Termo de Consentimento Livre e Esclarecido. Os dados foram coletados através de uma entrevista semiestruturada, realizada com cada familiar. Os familiares foram convidados a participar do estudo durante a atividade grupal, sendo agendado dia e hora para a coleta dos dados. As entrevistas foram realizadas em um consultório, através de um questionário com perguntas abertas e fechadas acerca da temática.

Foi realizada a análise temática dos $\operatorname{dados}^{(13)}$. Esta técnica possui três etapas: a pré-análise, a exploração do material e o tratamento dos resultados obtidos e interpretação. Na pré-análise realizou-se a transcrição literal das entrevistas e a elaboração das unidades de registro. Na fase de exploração do material, os dados foram codificados e agrupados por semelhanças e diferenças, gerando categorias e, na fase de tratamento dos resultados obtidos e interpretação, foram selecionadas as falas mais significativas, que foram discutidas a partir de estudiosos da temática.

Todos os preceitos éticos da resolução 196/96 do Conselho Nacional de Ética em Saúde foram levados em consideração ${ }^{(14)}$. O projeto foi encaminhado ao Comitê de Ética em Pesquisa na Área da Saúde (CEPAS) sendo aprovado através do parecer de número 113/2010. As falas dos familiares foram identificadas pela letra F, seguida do número da entrevista, como forma de garantir seu anonimato.

\section{RESULTADOS E DISCUSSÃO}

Os dados geraram duas categorias: Motivos que levaram os familiares a participarem do grupo de apoio/suporte no CAPS AD e Importância do grupo de apoio/suporte como estratégia de cuidado para o familiar cuidador.

\section{Motivos que levaram os familiares a participarem do grupo de apoio/ suporte no CAPS AD}

Verificou-se que o CAPS AD apresenta-se como uma alternativa de tratamento à internação no hospital psiquiátrico, possibilitando acesso aos profissionais da saúde e acompanhamento periódico no serviço.

Foi que ele estava doente e eu levei ele no psiquiatra e o médico, mandou ele para cá fazer acompanhamento no CAPS [...] na clínica $(F 7)$.

[...] a primeira vez ele mesmo se internou no Psiquiátrico. Ele me pediu para levar ele para se internar. Levamos ele e encaminhamos para o psiquiatra [...] ele já tinha se tratado aqui no CAPS com o doutor, só que ele não gostava do jeito dele. Ele foi para o Psiquiátrico e ficou vinte dias baixado. Depois ele veio encaminhado para cá e iniciou o tratamento $\left(F_{1}\right)$.

A família se constitui em um grupo, através do qual, os indivíduos iniciam seu primeiro contato com as normas e regras sociais. A princípio deveria proporcionar o suporte necessário para o desenvolvimento saudável, nortear os padrões de comportamento socialmente aceitos, os valores morais, sociais, éticos e espirituais. Em função da complexidade dos papéis parentais, ela vivencia conflitos múltiplos que, nem sempre possuem um desfecho positivo, levando a necessidade do redimensionamento de posturas diante das adversidades, nas quais se encontra submetida em busca da superação dos conflitos e da reorganização familiar ${ }^{(15)}$.

Em estudo que objetivou descrever o uso do grupo de apoio/suporte como estratégia para o acolhimento dos familiares dos pacientes internados em UTI e a avaliação dos participantes sobre o uso dessa estratégia para a satisfação das necessidades familiares de informação e suporte emocional evidenciou que o trabalho com grupos pode ser uma estratégia eficiente para a assistência de enfermagem, facilitando o atendimento de suas necessidades de informação, orientação e suporte psicológico ${ }^{(6)}$.

Frente à percepção do uso de drogas por seu familiar, a família passa a conviver com esta realidade e sofre por não saber lidar com os problemas ocasionados pela drogadição. Percebe-se que, muitas vezes, participa da atividade grupal por se sentir desamparada, acreditando que, assim, será ajudada a compreender o que é a dependência química e a manejar com o familiar dependente. 
Estava em desespero, sem saber lidar com ele. Vim aqui expliquei toda a situação. Me aconselharam a assistir à palestra que seria às três horas. Cheguei aqui e estava muito nervosa. Disseram já que a senhora está aqui precisando de ajuda, veio procurar ajuda para o seu filho e ele não está querendo vir. Ai eu falei tudo para elas e me disseram que ficasse para assistir o grupo para familiares. Achei muito bom (F9).

Compete aos profissionais da saúde apoiar a família, auxiliando-a a compreender e enfrentar o cotidiano que envolve cuidar do usuário de drogas. Assim, essa pode sair da condição de sentir-se responsável pelo uso de drogas do seu familiar para a construção de uma atitude positiva. No grupo, os familiares interagem com outros participantes vivendo situações semelhantes em um ambiente favorável à troca de experiências e esclarecimento de dúvidas quanto à situação vivida, ajudando a diminuir seu isolamento social ${ }^{(6)}$.

Tais mudanças começam a ocorrer, quando a família compreende a dependência química como doença e apoia com segurança e determinação o processo de cuidado desenvolvido pela equipe terapêutica $^{(16)}$. Estudo acerca de um grupo de mulheres que vivem e convivem com HIV/AIDS avaliou que o grupo favoreceu a resignificação da vida, pois nesse espaço se identificaram com os dilemas, dores e, também, discutiram alternativas para resolução de seus problemas ${ }^{(17)}$

Através da participação da família nos grupos de apoio no CAPS AD, os familiares referiram que aprenderam a lidar com o usuário de drogas fortalecendo-se para ajudá-lo, instrumentalizando-se para o seu cuidado. Acreditam que sem este apoio e o conhecimento adquirido nos grupos não conseguiriam auxiliá-lo na sua recuperação.

Para mim foi bom, porque não sabia lidar com ele, porque qualquer coisa eu explodia, dizia que iria chamar a polícia e internar ele, isso para mim foi uma coisa boa. Eu caí na real e consegui acalmar o meu filho (F9).

Vim para me fortalecer, para poder ajudar meu filho, porque a gente como família tem que estar preparado, porque se não, a gente não consegue (F4).

Para abordar Saúde Mental na família por meio de ações de educação em saúde faz-se necessário conhecê-la em seus múltiplos aspectos, oferecendo-lhe suporte. Dessa forma, para promover saúde mental na família torna-se primordial conhecer suas percepções e potencialidades ${ }^{(18)}$.
Apesar das diversas tentativas de tratamento, as recaídas são comuns e o abandono do tratamento também. Assim, as famílias têm sua participação nos grupos como forma de incentivar o usuário a manter-se em tratamento. A co-participação da família no tratamento apresenta-se como estímulo motivador, capaz de manter a força de vontade do paciente na sua recuperação. Percebem sua participação nas atividades grupais como chance que estão dando ao usuário de drogas para recuperar-se e manter-se inserido na família, como se essa aderência fosse considerada uma condição para a reinserção social deste paciente.

Vim para trazer ele, porque não vem sozinho. Tenho que andar com ele. Toda vez que ele vem, eu venho junto $(F 7)$.

Um motivo maior. Uma ajuda para ele e para mim, porque acabo participando dos grupos e porque dessa vez ele está com força de vontade. Dei uma chance, ele ouviu falar daqui e eu disse, vou contigo. A enfermeira me ajudou e estamos aqui (F5).

Percebem que a participação nos grupos do CAPS AD é uma possibilidade de receber o apoio não só, dos profissionais da saúde, mas, também, de outros familiares que vivenciam as mesmas situações, medos e incertezas, acreditando serem compreendidas, tendo o sentimento de pertença ao grupo.

É que o meu filho é dependente químico. Eu vim até aqui para pedir um apoio a todas as pessoas que, como eu, vivem esta situação. Eu me sinto muito bem de estar com eles. Me entendem. Gosto e pretendo continuar $(\mathrm{F} 2)$.

Dentre as estratégias de tratamento oferecidas, o grupo para dependentes químicos e/ou seus familiares apresenta-se como instrumento de intervenção, inserido na rede de suporte sempre levando em consideração suas potencialidades: a oportunidade de fazer amizades, receber acompanhamento multiprofissional e medicamentoso, conhecer realidades semelhantes às suas; interagir com outras pessoas, ser fonte de informações e referência de cuidado como um todo ${ }^{(18)}$.

Estudo acerca do grupo como suporte a familiares de crianças internadas em UTI concluiu que através do mesmo foi possível fornecer informações e orientações de interesse comum para vários familiares ao mesmo tempo avaliando a atividade 
grupal como uma estratégia para o uso racional do tempo e esforço dos enfermeiros. Para os familiares, a participação em um grupo de pessoas vivendo situações semelhantes pode ser uma experiência com valor terapêutico, tanto pelo suporte recebido dos outros participantes, como pela oportunidade de partilhar sua própria experiência e dar suporte a outras pessoas ${ }^{(6)}$.

\section{Importância do grupo de apoio/ suporte como estratégia de cuidado para o familiar cuidador}

Os familiares referem que a participação no grupo é importante porque este se apresenta como fonte de escuta, no qual se sentem acolhidos, o que lhes permite falar, chorar, conversar, pedir ajuda, ou seja, expressarem seus sentimentos frente à vivência da dependência química, sem medo de serem julgados, apresentando-se como um importante recurso terapêutico.

Só em escutar, ouvir a gente já é uma ajuda, porque tem muita gente que não quer escutar, não tem nada a ver com a tua vida. A gente está vindo para cá. Eles escutam a gente $(F 8)$.

Para mim é bom, porque vejo que não estou sozinha, porque posso vir, chorar, conversar, pedir ajuda, sei que aqui eu encontro ajuda (F3).

A participação em grupos de apoio promove a troca de experiências com outras pessoas com problemas semelhantes, o que lhes possibilita a constatação de que não estão sozinhas. A oportunidade de compartilhar problemas entre si é uma forma de se sentirem incluídas no grupo, apoiadas, manterem a autoestima, acreditar, confiar e superar algumas dificuldades ${ }^{(19)}$. Fazer parte de grupos de apoio pode ser considerado como fonte de suporte social, ao se perceber que os grupos funcionam como sustentáculo para o processo de recuperação e adaptação à nova condição, e ainda como ambiente de transformação psicofísico e psicossocial $^{(19)}$.

O uso de drogas ainda é percebido na sociedade como algo marginal e o usuário de drogas se apresenta como alguém que possui uma importante falha de caráter. Por isso, tanto este, como sua família são estigmatizados e sofrem preconceito. A participação no grupo auxilia a família a compreender a dependência química como uma doença.
Meu tratamento é para desabafar. Porque tem muita gente que não entende que o alcoolismo é uma doença. Acham que é sem-vergonhice e falta de vergonha na cara e é uma doença. Agora eu entendi isso (F1O).

Através da participação nos grupos de apoio, os familiares trocam experiências aprendendo a lidar com as situações cotidianas. Sentem-se aliviados podendo ver que seu problema frente ao que é enfrentado por outras famílias adquire outra dimensão, podendo, até mesmo, parecer mais fácil e possível de ser enfrentado. Após as atividades grupais parecem sentir-se renovados e fortalecidos.

O grupo é um alívio, porque tu escutas os outros. O que está acontecendo na vida dos outros. O que está acontecendo na tua. É maravilhoso. Tu sai com outra expectativa e acabas torcendo para os outros também (F5).

Vemos histórias de pessoas que o teu problema que parece tão grande, de repente se torna pequeno, perto de depoimentos de mães, do desespero de pessoas. Tu vê que tens problemas, e que tem gente que vive numa situação pior que a tua. Tu sai renovada daqui, cria forças! (F4).

Ao avaliar a eficiência da atividade grupal evidenciou-se que o grupo apresenta-se como uma estratégia útil para manter a força e esperança entre as famílias participantes, fornecer espaço e ambiente propícios à aprendizagem e compartilhamento de informações e se configurar em uma rede de apoio para essas pessoas. Por meio da oferta de informações e suporte emocional é possível ajudar as famílias a enfrentar a crise vivida, atenuar seu sofrimento e reduzir a ansiedade ${ }^{(20)}$.

A participação no grupo apresenta-se como uma fonte contínua de recebimento de informações, que mantém a família fortalecida auxiliando-a a tomar decisões relativas ao cuidado do usuário de drogas, respaldada no conhecimento recebido.

Tem coisas que ainda estou na dúvida, porque ele foi para a fazenda e retornou, porque deu recaída. E eu não sabia como lidar com ele. Fazia perguntas e as pessoas iam me dizendo. Tinha medo que ele pegasse de alguém. Então, dava o dinheiro para ele usar. Eu me obrigava a dar, sabendo que não era para dar e não era do meu gosto também (F2).

Os processos de interação entre profissionais e usuários, no contex to da saúde, têm sido pautados por concepções que permaneceram sedimentadas na tradição de um profissional portador do saber se 
relacionando com um usuário alienado deste saber, com o intuito de educá-lo para um melhor comportamento em saúde. No entanto, levar em consideração o conhecimento dos usuários e familiares acerca de sua problemática amplia as possibilidades terapêuticas do grupo, pois direciona as estratégias assistenciais, possibilitando que sejam mais efetivas.

Referem que a participação continuada nos grupos apresenta-se como periódico provimento de energias e força fundamental para manter o familiar cuidador amparado e motivado a ajudar o dependente a continuar seu tratamento, servindo de alicerce à sua recuperação.

Eu marco e elas me esperam. Tem psicóloga e psiquiatra. $O$ atendimento que geralmente precisamos, para aguentar o tranco, que não é fácil (F3).

Estar ouvindo o depoimento de outras mães, vendo a realidade, como o que conversamos com eles, porque estou sempre conversando com ele, e converso muito com a menina. Tem uma menina que eu crio, ela tem dezessete anos. Acho muito bom. Esse grupo está me dando muita força para enfrentar, porque às vezes, dizem que tem recaída $(F 1)$.

Neste sentido, observa-se a necessidade de capacitação e aperfeiçoamento constante dos profissionais, a fim de que ocorra a articulação entre as abordagens utilizadas no grupo, para que os pacientes mantenham-se motivados e instigados a participarem dos grupos de apoio.

\section{CONSIDERAÇÕES FINAIS}

O estudo evidenciou o papel da família como principal fonte de apoio e sustentação, sendo o alicerce que mantém o vínculo social do usuário de drogas. Acredita-se que é fundamental a atuação compartilhada entre profissionais e familiares para a efetividade do seu tratamento.

Tendo em vista o papel central da família na recuperação do usuário de drogas esta precisa ser considerada como uma parceira, mas também ser reconhecida como co-dependente, portadora de adoecimento decorrente de suas experiências traumáticas e vivências cotidianas impostas pelo usuário de drogas. Como co-dependente precisa de acompanhamento terapêutico para aprender a lidar com estas situações e poder atuar junto a seu familiar usuário de drogas, de forma mais instrumentalizada, não permitindo que ele interfira em suas expectativas e projetos de vida.
Conclui-se ser o grupo de apoio/suporte uma importante estratégia de cuidado aos familiares de usuários de drogas, apresentando-se como ferramenta a ser utilizada na promoção do cuidado prestado, em especial pelo enfermeiro, com vistas à realização da educação em saúde, da prevenção, promoção e recuperação da saúde de indivíduos e grupos sociais. É uma ferramenta de atenção em saúde a ser utilizada pelo enfermeiro que precisa dominar esta tecnologia com vista a tornar seu trabalho no CAPS AD produtivo.

Assim, como os outros profissionais do CAPS AD, o enfermeiro deve instrumentalizar-se para realizar esta atividade no sentido de dar conta da integralidade do cuidado. O uso do grupo de apoio/suporte como recurso terapêutico pode colaborar para a construção de uma prática assistencial humanizada e acolhedora. Entende-se o grupo, apenas como uma das estratégias utilizadas pelo enfermeiro no seu fazer cotidiano. No entanto, por seus efeitos positivos recomenda-se que os enfermeiros utilizem esta tecnologia como importante estratégia para a assistência de enfermagem à família do usuário de drogas atendido no CAPS AD.

\section{REFERÊNCIAS}

1 Aguilar LR, Pillon SC. Percepción de tentaciones de uso de drogas en personas que reciben tratamiento. Rev Latino-Am Enferm. 2005;13(n.spe):790-7.

2 Canoletti B, Soares CB. Programas de prevenção ao consumo de drogas no Brasil: uma análise da produção científica de 1991 a 2001. Interface Comun Saúde Educ. 2005; 9(16):115-29.

3 Organização das Nações Unidas. Relatório Mundial sobre drogas 2010 [Internet]. 2010 [citado 2011 set 09]. Disponível em www.onu.org

4. Delfini PSS, Sato MT, Antoneli PP, Guimarães POS. Parceria entre CAPS e PSF: o desafio da construção de um novo saber. Ci Saúde Col. 2009;14(1):1483-92.

5 Loomis ME. Group process for nurses. Saint Louis: Mosby; 1979.

6 Oliveira LMAC, Medeiros M, Barbosa MA, Siqueira KM, Oliveira PMC, Munari DB. O acolhimento de familiares de pacientes internados em UTI: a tecnologia de grupo como estratégia para o cuidado de enfermagem. Rev Esc Enferm. USP. 2010; 44(2):429-36. 
7 Moraes LMP, Lopes MVO, Braga VAB. Componentes funcionais da teoria de Peplau e sua confluência com o referencial de grupo. Acta Paul Enferm. 2006;19(2):228-33.

8 Montrone AVG, Fabbro MRC, Bernasconi PBS. Grupo de apoio/ suporte à amamentação com mulheres da comunidade: relato de experiência. Rev APS. 2009; 12(3):357-62.

9 Klein MMS, Guedes CR. Intervenção psicológica a gestantes: contribuições do grupo de suporte para o promoção da saúde. Psicol Ci Prof. 2008;(28)4:862-71.

10 Filzola CLA, Tagliaferro P, Andrade AS, Paravini SCI, Ferreira NMLA. Alcoolismo e família: a vivência de mulheres participantes do grupo de autoajuda Al-Anon. J Bras Psiquiatr. 2009;58(3):181-6.

11 Almeida VCF, Lopes MVO, Damasceno MMC. Teoria das relações interpessoais de Peplau: análise fundamentada em Barnum. Rev Esc Enferm. USP. 2005;39(2):202-10.

12 Triviños ANS. Introdução à pesquisa em ciências sociais: a pesquisa qualitativa em educação. São Paulo: Atlas, 2009.

13 Minayo MCS. Pesquisa social: teoria, método e criatividade. $29^{\mathrm{a}}$ ed. Petrópolis: Vozes, 2010.

14 Ministério da Saúde (BR). Portaria n 196/96: dispõe sobre diretrizes e normas regulamentadoras de pesquisa com seres humanos. Brasília (DF): Conselho Nacional de Saúde; 1996.

15 Macêdo VCD, Monteiro ARM. Educação e saúde mental na família: experiência com grupos vivenciais. Texto \& Contexto Enferm. 2006;15(2):222-30.

16 Hermeto EMC, Sampaio JJC, Carneiro C. Abandono do uso de drogas ilícitas por adolescente: importância do suporte familiar. Ver Baiana Saúde Públ. 2010;34(3):639-52.

17 Santos W, Munari DB, Medeiros M. O grupo de mulheres que vivem e convivem com HIV/ AIDS: um relato de experiência. Rev Eletr enferm. 2009; $11(4): 1043-8$.

18 Souza J, Kantorski LP, Vasters GP, Luis MAV. Rede social de usuários de álcool, sob tratamento, em um serviço de saúde mental. Rev Latino-Am. Enferm. $2011 ; 19(1): 140-7$.

19 Pinheiro CPO, Silva RM, Mamede MV, Fernandes AFC. Participação em grupo de apoio/ suporte: experiência de mulheres com câncer de mama. Rev Latino-Am Enferm. 2008;16(4):733-8.

20 Santos LF, Oliveira LMAC, Munari DB, Peixoto MKAV, Silva CC, Ferreira ACM et al. Support group as a strategy for nursing care for the families of hospitalized newborns. Rev Eletr Enferm. 2012;14(1):42-9.

\author{
Endereço do autor / Dirección del autor / \\ Author's address: \\ Daiani Modernel Xavier \\ Av. Pelotas, 1443, Rural \\ 96211-002, Rio Grande, RS \\ E-mail: daiamoder@ibest.com.br
}

